Investigaciones Fenomenológicas, n. 9, 2012, 383-405.

e-ISSN: $1885-1088$

\title{
El CARTESianismo de Richir. Aproximación A LA "Tercera meditación fenomenológicA"
}

\author{
Sacha Carlson \\ Université de Louvain-la-Neuve, Bélgica \\ sachacarlson@gmail.com
}

\begin{abstract}
Resumen: Este artículo trata de poner en claro el estatuto del "cartesianismo" de Richir en su tercera Meditación fenomenológica, en la cual Richir propone una nueva versión de la reducción fenomenológica. Mostraremos en primer lugar cómo Richir centra su lectura de Descartes sobre el carácter hiperbólico de la duda, descubriendo en él un momento propiamente fenomenológico. Examinaremos luego el modo en el que Richir ensaya un acercamiento del cogito cartesiano respecto de la concepción heideggeriana del ser-para-la-muerte. Por último, explicaremos cómo Richir critica esta "vía cartesiana" ( $y$, por ende, también heideggeriana) para elaborar su propia concepción de la facticidad.
\end{abstract}

Palabras clave: Richir, fenomenología, fenómenos, Meditaciones fenomenológicas, Descartes, Heidegger, reducción, Epojé, hipérbole, facticidad.

\begin{abstract}
This article proposes to study the status of Richir's cartesianism in his third Méditation phénoménologique, where a new approach to phenomenological reduction has been suggested. As a beginning, we will show how Richir focuses his reading of Descartes on the hyperbolic nature of the doubt, and detects in the text a neatly phenomenological moment. Then, we will study the ways Richir gets closer the Cartesian cogito and the Heidegger's concept of Being-towards-death. And finally, we will explain how Richir critises the Cartesian (and Heideggerian) way" in order to develop his own conception of the facticity.
\end{abstract}

Keywords: Richir, phenomenology, phenomena, Phanomenological Meditations, Descartes, Heidegger, reduction, Épochè, hyperbol, facticity.

\section{Como sabemos, la tercera de las Meditaciones fenomenológicas ${ }^{1}$ de Marc} Richir, dedicada la cuestión de la reducción fenomenológica, lleva como subtítu-

\footnotetext{
${ }^{1}$ Marc Richir, Méditations Phénoménologiques. Phénoménologie et phénoménologie du langage, Grenoble, Jérôme Millon, 1992 (que citaremos de ahora en adelante por las siglas MP). La tercera Meditación (pp. 67-134) se titula: "Para una epojé fenomenológica hiperbólica. Meditación hipercartesiana".

Para una presentación global, en español, del pensamiento de M. Richir, podemos remitirnos a: Pablo Posada Varela, "En torno a la singladura filosófica y fenomenológica de Marc Richir".pdf, Eikasia 40 (2011) 239-290. Para la cuestión, más específica (y afín a este trabajo) de la epojé hiperbólica y del "hipercartesianismo" de Richir, puede consultarse el apartado titulado "La cuestión de la hipérbole y la recuperación de Descartes" (pp. 448-453) del artículo de Pablo Posada Varela "Hipérbole y concretud en parpadeo: en torno al último libro de Marc Richir "Variaciones sobre el sí mismo y lo sublime".pdf, Eikasia 34 (2010) 439-458), así como el apartado 3.4. titulado "Reconsideraciones sobre la epojé fenomenológica hiperbólica" (pp. 417-419) del artículo "Introducción a Variaciones II y a otros aspectos de la fenomenología de Marc Richir".pdf, Eikasia 40 (2011) 357-442. Por último, para un tratamiento detalla-
} 
Io: "Meditación hipercartesiana". Tan sorprendente como digno de reseñar es que Richir elija a Descartes como interlocutor privilegiado. En el $\S 1$ de esta Meditación parece como si Richir pusiese en juego a Descartes frente a Husserl al tiempo que lo sitúa del lado de Heidegger. Para empezar, se habrá de conceder que, al menos de primeras, se antoja cosa extraña, en fenomenología, "preferir" a Descartes respecto de Husserl: Husserl, como sabemos, si bien le reconoce a Descartes el mérito de haber dirigido su atención filosófica sobre el cogito y sobre el ego sum, no por ello dejaba de reprocharle al filósofo francés el haber "marrado la orientación transcendental"2 de la fenomenología. Se pregunta, pues, uno qué es lo que Descartes, el Descartes incontestablemente metafísico, puede aportar respecto de Husserl, o puede aportar, más allá de Husserl, a la fenomenología en general.

Por otro lado, no deja de ser extraña esa intención de leer a Descartes junto a Heidegger cuando bien sabemos hasta qué punto, hasta qué extremos, rayanos en la obsesión, se esforzará el autor de Sein und Zeit durante toda su andadura en "desmarcarse" de Descartes ${ }^{3}$. Pues bien, quisiera adelantarlo de entrada: lo que, y ello al mayor extremo, interesa a Richir en Descartes reside, obviamente, en el carácter hiperbólico de la duda, duda hiperbólica sistemáti-

do de la cuestión de la epojé hiperbólica y de la reducción fenomenológica en clave mereológica, cfr. Pablo Posada Varela, "Concrétudes en concrescences. Éléments pour une approche méréologique de la réduction phénoménologique et de l'époché hyperbolique", Annales de Phénoménologie 10 (2011).

Pueden consultarse, por lo demás, otros artículos y algunos libros de Richir en la página: www.laphenomenologierichirienne.org

${ }^{2}$ Por lo que hace al homenaje, tenemos, por ejemplo: "Que el ego sum o el sum cogitans deban ser vistos como apodícticos, luego que con ello hollemos un dominio de ser apodíctico y primero es cosa que Descartes, como sabemos, ya supo ver. Subraya, en efecto, el carácter indubitable de esta proposición y afirma claramente que incluso el "yo dudo" ya supone el 'yo soy'. Se trata, también en su caso, del yo que se capta a sí mismo [qui se saisit lui-même], tras haber puesto fuera de validez el mundo empírico al ser éste susceptible de duda" (Meditaciones cartesianas, § 9). El § 10 estigmatiza, por el contrario, una "tendencia" en Descartes, la de "considerar" el ego cogito como un "axioma" apodíctico, axioma que, unido a otros aún no desvelados, o incluso a hipótesis halladas por vía inductiva, ha de servir de fundamento a una ciencia 'deductiva' y explicativa del mundo, ciencia 'nomológica' que procede ordine geometrico, análoga, precisamente, a las ciencias matemáticas". De hecho, esta crítica de Husserl a Descartes parte de una antigua dificultad - Cfr. las Respuestas a las terceras objeciones - de lectura del texto cartesiano: ¿es el cogito el primer elemento de una cadena deductiva, tal y como podría llevarnos a pensar la expresión cogito ergo sum- expresión que nos encontramos, por ejemplo, en el Discurso del método, pero no en las Meditaciones metafísicas -expresión que, efectivamente, tiene el aspecto de un silogismo? Si es el caso, entonces no puede ser el fundamento transcendental que busca Husserl: así, el $\S 10$ se titula: "De cómo Descartes marró la orientación transcendental". La lectura que propone Richir consiste precisamente en mostrar que el momento del cogito cartesiano es el de una experiencia fenomenológica.

${ }^{3}$ Jacques Taminiaux ha mostrado con mucho tino cómo Heidegger, desde Sein und Zeit hasta su obra sobre Nietzsche, siempre ha buscado desmarcarse de Descartes al tiempo que buscaba, por otro lado, reapropiarse otros autores tales como Aristóteles, Kant, Hegel, o Nietzsche (cfr. "D'une double lecture de Descartes", en Lectures de l'ontologie fondamentale. Essais sur Heidegger, Grenoble, J. MilIon, 1989, pp. 213-230). El autor recuerda cómo Heidegger indica varias veces que el desacuerdo entre él y Husserl se debía, en su mayor parte, al cartesianismo del que Husserl se reclamaba. 
camente puesta en juego en las Meditaciones Metafísicas ${ }^{4}$ pero que apenas encuentra uno -o acaso no encuentre en absoluto- en Husserl ${ }^{5}$; y también será la hipérbole lo que le permita a Richir ensayar ese acercamiento, casi "irónico" ${ }^{\prime \prime}$, entre Heidegger y Descartes ya que, como veremos, en la punta última de la hipérbole, es decir, antes del análisis del trozo de cera, el cogito cartesiano en cierto modo aparecerá como aquello que Heidegger, por su lado, piensa como la facticidad del Dasein ${ }^{7}$.

\section{LA RECUPERACIÓN RICHIRIANA DE LA DUDA HIPERBÓLICA CARTESIANA}

¿Qué decir, pues, en primer lugar, de Descartes? ${ }^{8}$. Se antoja inútil recordar aquí cómo y por qué Descartes, en sus Meditaciones, trata de "establecer algo que sea firme y constante en las ciencias" ${ }^{\prime \prime}$ y que esta empresa empieza por lo que se ha denominado "duda metódica", relativa a todo lo que es "recibido como creencia [reçu en créance]", todo aquello a lo que otorgamos crédito, es decir, en primer lugar, los sentidos y las sensaciones, que son aquellos casos en los que la creencia, el crédito otorgado, parece tener mayor pregnancia: ¿acaso hay algo más disparatado que dudar de que esté yo aquí y ahora, con mi cuerpo y las cosas que me rodean? Me fijaré más bien en el modo en el que Richir rastrea e interpreta, desde las primeras páginas del texto de Descartes,

\footnotetext{
${ }^{4}$ Cuando nos refiramos aquí a Descartes, haremos alusión a las Meditaciones. En el prefacio a sus propia Meditaciones, Richir señala por lo demás: "En este camino nos hemos encontrado con dos momentos propiamente instauradores para la fenomenología: las 'Meditaciones metafísicas' de Descartes y las 'Meditaciones cartesianas' de Husserl. [...] Nos hallamos, claro está, lejos del Descartes de la ciencia instituida, pero cerca, e incluso muy cerca, del Descartes de la duda hiperbólica". MP, p. 7.

${ }^{5}$ En Descartes, la duda se lleva hasta la hipérbole "como jamás lo hará el propio Husserl de las Meditaciones cartesianas a no ser de modo muy fugitivo, apenas si por ráfagas, en muy contadas ocasiones". MP, p. 78.

6 "Esto equivale, por lo que hace a Heidegger, a hacer estallar el solipsismo existencial de la Jemeinigkeit consectaria del mismo, o también, por hablar de modo más 'abstracto', a 'radicalizar' el cartesianismo heideggeriano - nos hacemos plenamente cargo de la ironía de la fórmula que aquí avanzamos $[\ldots]^{\prime \prime}$. MP, p. 88; el subrayado es mío.

7 La dificultad estará en comprender la diferencia entre lo que Richir llama facticidad, incluso cuando se reclama de Heidegger, y lo que el propio Heidegger entiende bajo el mismo término.

${ }^{8}$ Aparte de la tercera Meditación de Richir (en particular las pp. 78-84), me apoyo aquí también en los dos textos siguientes, en los cuales Richir también sigue el decurso de las Meditaciones metafísicas: el artículo, escaneado y disponible en pdf con "cliquear" sobre el vínculo que a continuación se ofrece, "Doute hyperbolique et machiavélisme. Institution du sujet moderne chez Descartes".pdf (publicado en 1997 en los Archives de philosophie, vol. 60, 1 (1997) 109-122; y, en la obra Phantasia, imagination, affectivité. Phénoménologie et anthropologie phénoménologique, Grenoble, J. Millon, 2004, pp. 131151(a esta obra haré alusión, a partir de ahora, con las siglas "PIA").

9 Descartes, Meditaciones metafísicas, p. 13. Utilizo el texto tal y como está publicado, en francés y en latín, Paris, P.U.F., 1992. La paginación citada es la que viene tradicionalmente usándose, a saber, la del tomo IX de la edición Adam et Tannery. A partir de ahora, con las siglas: MM.
} 
lo que ha dado en llamarse "duda hiperbólica"10, es decir, radical, y que parece jugar como en contrapunto a la duda metódica.

Efectivamente, la hipérbole ya está presente desde las primeras páginas del texto. Lo está bajo esos dos semblantes que son la locura y el sueño. En primer lugar, "la punta extrema de la hipérbole"11 aparece muy pronto en el texto, ya con la hipótesis de la locura: no puedo razonablemente dudar de que estoy aquí, con mi cuerpo y las cosas circundantes "a no ser que -escribe Descartes- me compare con eses insensatos [...] [que] aseguran una y otra vez ser reyes cuando, en realidad, son muy pobres $[\ldots]^{11^{12}}$. A lo que Descartes repone, en una célebre réplica: "¿Y qué? Se trata de locos, y no sería yo menos extravagante si me dejase guiar por su ejemplo"13. ¿Cómo entender esta revocación de la locura, a propósito de la cual tantísimo se ha glosado ${ }^{14}$. No se trata, considera Richir, de una revocación o de un reprimir [refoulement] sino antes bien de una restricción metódica: "para Descartes, efectivamente, la locura carece de hilo conductor, es decir [...] nadie decide jamás ni puede decidir, al albur de una simple variación ${ }^{15}$, volverse loco $^{16}$ y ésa es la razón por la que la verdadera locura no puede, metódicamente, tomarse en consideración.

De la locura pasamos a otra figura de la hipérbole, la de esa "pequeña locura" que es el sueño. Efectivamente, me acontece, por veces, creer que estoy despierto cuando, sin embargo, resulta que estoy durmiendo, y es por ello por lo que tengo todo el derecho a preguntarme si no será que estoy durmiendo a pesar de creer estar despierto. Se trata, en definitiva, de preguntarse-como lo hará el propio Husserl más tarde- si no se limitará el mundo a no ser sino mero

\footnotetext{
${ }^{10}$ El propio Descartes no hace un uso sistemático de la expresión "duda hiperbólica", en todo caso, no en el texto mismo de las Meditaciones. Escribe sin embargo, en la penúltima página de su texto: "Y debo rechazar todas las dudas de estos días pasados por hiperbólicas (hyperbolicae) y ridículas, particularmente esa incertidumbre tan general tocante a un sueño que no podría distinguir de la vigilia [...]". MM, p. 42. Esto ha dado pie a los comentadores para designar como hiperbólico este momento radical de la duda en el que no se trata ya de sospechar de un error del juicio o de los sentidos (ése es la duda metódica), sino de sospechar del sentido mismo del ser y del no ser de la realidad, como sucede con el argumento del sueño o del Genio Maligno. Para Richir, claro está, la duda "hiperbólica", inada tiene de "ridícula"!

11 PIA, p. 132.

12 MM, p. 14.

${ }^{13} \mathrm{Idem}$.

${ }^{14} \mathrm{Cfr}$. lo que ha dado en llamarse la "querella o disputa de la locura [la querelle de la folie]", que opuso a Michel Foucault y a Jacques Derrida. Un buen panorama de esta cuestión disputada, así como las referencias esenciales, se encuentra en: Denis Kambouchner, Les méditations métaphysiques de Descartes. Introduction générale. Première méditation, Paris, P.U.F., 2005, pp. 381-394.

${ }^{15}$ En el sentido husserliano de la variación eidética. [NdT]

16 PIA, p. 131.
} 
"sueño coherente". El argumento de Descartes para formular esta hipótesis consiste, al cabo, en aducir que no existe criterio alguno intrínseco a los sentidos y a los sensibles que permita distinguir, en la sensibilidad, el modo imaginativo del modo perceptivo ${ }^{17}$. Así, Descartes, por cuanto busca asentar la ciencia como saber objetivo, se verá obligado a revocar como dudoso todo lo que es intuitivamente figurable: por lo tanto, tanto lo real como lo imaginario. De ahí que, en este punto del recorrido, sólo las matemáticas parezcan conservar su validez: "tanto si estoy despierto como si duermo, dos más tres siempre formarán el número cinco"18. Pero también será este último campo de validez de las cosas el que habrá de quedar, de igual modo, en suspenso, y ello mediante la puesta en juego, sistemática esta vez, de la duda hiperbólica.

Es lo que se llevará a cabo, como sabemos, con la entrada en escena de Dios:

[...] Hace tiempo que albergo en mi mente cierta opinión, a saber, que hay un dios que todo lo puede, y por el cual he sido creado y producido tal y como soy. Ahora bien, ¿quién puede asegurarme que ese dios no haya obrado de tal modo que no haya ni tierra ni cielo alguno, y ningún cuerpo extenso o figura, ninguna magnitud ni lugar y que, con todo, tenga yo los sentimientos de todas estas cosas, y que todo ello no me parezca existir de otro modo que como precisamente lo veo ${ }^{19}$

Mientras que la duda metódica trataba sobre el riesgo factual del error, la duda hiperbólica, cuando se pone sistemáticamente en juego, se hace consectaria de una sospecha de engaño: podría ocurrir que algún Otro se las estuviera arreglando para que yo, de hecho, percibiese todas estas cosas (la tierra, el cielo, etc.) cuando, en rigor, resulta que no existen en absoluto. O podría suceder también que el mundo, e incluso las matemáticas, no sean más que un mero sueño, una ilusión coherente. Es precisamente esta sospecha la que, de hecho, articula duda hiperbólica y duda metódica -además de equivocarme [me tromper], también puedo, aun poniendo todo el cuidado del mundo en no equivocarme, resultar engañado [être trompé] por una fuerza omnipotente- lo cual me impide acordarles crédito [accorder créance] a las cosas del mundo e incluso a las matemáticas, a todo lo que, en suma, parece más consolidado, es de-

\footnotetext{
${ }^{17}$ Cfr. PIA, p. 132.

${ }^{18}$ MM, p. 15.

19 MM, p. 16.
} 
cir, a todo aquello que Richir engloba bajo el concepto de "institución simbólica" ${ }^{\prime 20}$.

La dificultad de ese tipo de duda, cercana a una epojé escéptica, reside, claro está, en su carácter enteramente teórico: por mucho que me imponga dudar en teoría de todo aquello a lo que otorgo crédito, en la práctica, la institución simbólica siempre acabará por alcanzar mi radicalidad para acabar teniendo vigencia en los asuntos humanos de tal suerte que respecto de todas esas cosas puestas en duda "mucho más sensato es creerlas que negarlas" ${ }^{21}$. Precisamente por ello, la duda hiperbólica ha de ir más lejos: es preciso "que ponga todo cuidado en engañarme a mí mismo" (ipsum fallam) fingiendo de forma activa que "todos esos pensamientos son, por algún tiempo (aliquandiu) simplemente (omnino) falsos e imaginarios" ${ }^{\prime 22}$. Sea como fuere, todos esos pensamientos, quiéralo o no, permanecen verosímiles por cuanto proceden de la institución simbólica; la epojé sólo será de veras hiperbólica si, por verosímiles que se me antojen, hago como si fueran "falsos e imaginarios"; así, escribe Richir:

La fábula o la ficción sólo se sustenta en un fingimiento [feinte], suerte de semblante activo $^{23}$, un hacer como si que es ya del todo distinto de la puesta en suspenso escéptica. Soy pues yo el encargado de elaborar el supuesto de "un determinado Genio Maligno, no menos astuto y embustero que poderoso, que ha empleado toda su industria en engañarme". Se debe, pues, "a mi poder, el suspender mi propio juicio", y es precisamente así como "hasta tal punto habré preparado mi mente ante todas las astucias de este gran engañador que, por poderoso y astuto que sea, jamás podrá imponerme nada". ${ }^{24}$

De este modo, sólo si soy yo quien toma la iniciativa de hacer, activamente, "como si" un Genio Maligno emplease toda su industria en engañarme, puedo desbaratar toda posible engañifa. Y por ello mismo insiste Richir en que todo el movimiento de la duda hiperbólica reposa, al fin y al cabo, en la libertad del sujeto, libertad que "asoma o da la cara [paraît], en un primer momento, como libertad de la epojé, de la suspensión del juicio"25. En este sentido, sí es cierto

${ }^{20}$ Cfr. PIA, pp. 133 s.

${ }^{21} \mathrm{MM}$, p. 17.

${ }^{22}$ Idem.

${ }^{23}$ Aquí Richir retoma un concepto parejo al concepto de "semblant" y de "semblant solide", conceptos centrales en la filosofía (muy marcada por la fenomenología, por no llamarla fenomenológica tout court) del que fuera su gran amigo Jean-Toussaint Desanti. [NdT].

24 "Doute hyperbolique et machiavélisme. Institution du sujet moderne chez Descartes".pdf (paru en 1997 dans les Archives de philosophie), p. 113.

25 Ibidem, p. 114. 


\section{que cabe oír resonar, en las Meditaciones de Descartes, como el eco de una reflexión teológico-política de segundo grado sobre la libertad del hombre ${ }^{26}$. Con todo, es preciso preguntarse antes por el sentido fenomenológico del Genio}

Maligno:

Cabe entonces que nos preguntemos qué articula esta operación [i.e. la operación del fingimiento o ficción [feinte] con la suposición del Genio Maligno (genius malignus). ¿Ocurrirá acaso que no sea éste sino yo mismo, pero bajo una forma "externa"? ¿Cómo, efectivamente, puedo fingir, imaginar (fingere; fingieren o umfingieren diría Husserl) que todo esto que "creo" es falso e imaginario? ¿Acaso se trata aún, propiamente, de mí? En este punto hemos de permanecer atentos al hecho de que la ficción ya no se refiere a los contenidos, que permanecen iguales, sino a su valor como posibles verdades, a su valor de verdad ${ }^{27}$. Así pues, no es que esté imaginando algo más allá de lo real, algo suplementario y errado, sino que, llevado por la sospecha del engaño, doy en imaginarme que no es por mor de un "crédito [créance]" (doxa) distinto al que tiene vigencia en la imaginación (véase el primer ejemplo del sueño) por lo que, ahora, otorgo crédito a mis pensamientos. Es, por lo tanto, como si los contenidos supuestamente soñados ( $y$ ficticios) me viniesen, en el suspenso (epojé) de mi "crédito [créance]", de un lugar otro [ailleurs] ${ }^{28}$, por lo que, en definitiva, es como si absolutamente nada aparte de este "crédito" y mi suspensión fuese de veras cosa mía. ${ }^{29}$

${ }^{26}$ Cfr. MP, p. 80: se trata, de hecho, del hilo conductor del importante artículo sobre duda hiperbólica y "maquiavelismo", op. cit.

${ }^{27}$ La expresión "valor de verdad", usada por el autor del artículo así como por el propio Richir puede resultar algo confusa. Tratemos de encaminar el sentido en el que ha de entenderse. No se trata del "valor de verdad" en sentido lógico, lo cual supondría, precisamente, volver a la cuestión de la duda metódica y del error. Se trata más bien del valor de esos contenidos de pensamiento no tanto en su contenido cuanto, antes bien, en su pretensión de verdad, en su pertinencia como posibles verdades (o falsedades). Dicho de otro modo: en régimen de duda hiperbólica, la falsedad no radicaría en una no adecuación, sino antes bien en el origen de la facticidad de ese mismo pensamiento, en haber sido enteramente simulado, montado por completo, junto a mi impresión de ser, tal pensamiento, pensamiento mío. Incluso esto último sería algo que la omnipotencia del Genio Maligno podría haber producido, generado, "mandándome" tal pensamiento junto a su apariencia o fenómeno de ser experiencia o pensamiento -o, en suma, "pensar" en el sentido amplio que entiende Descartes- míos. Así las cosas, y puesta en juego la duda hiperbólica, no tiene sentido volver a tratar los contenidos de pensamiento en términos de duda metódica o siquiera de duda. No se trata ya de escrutar su contenido. El problema se desplaza al origen mismo de su facticidad como pensamientos, y a la cuestión de quién sea el verdadero titular de estos pensamientos: sin antes enfrentar este primer problema, relativo a la pretensión de verdad o "valor de verdad", de ser "posibles verdades" o siquiera "poder ser verdades" -así no lo sean de hecho- de estos pensamientos, no tiene sentido esmerarse en cotejar el detalle veritativo de su contenido. Dicho de otro modo: un pensamiento falso mío tiene más "valor de verdad" (en el sentido en que lo emplean aquí tanto Richir y Sacha Carlson) que un supuesto juicio verdadero que, sin embargo, me hubiese sido "montado", "armado de cabo a rabo" por un Genio Maligno omnipotente. Por "verdadero" que, de hecho, resulte, no es un verdadero pensamiento (mío), su "valor de verdad" o "valor como (posible) verdad" queda, por así decirlo, desbaratado de antemano: se trataría de un falso pensamientoverdadero, generado por un falso pensar o una apariencia o simulacro de pensar o de "yo pienso" (pues el verdadero "pensar" es un pensar en primera persona). Lo que en este contexto se entiende o sobreentiende aquí por "verdadero pensamiento" no tiene, precisamente, nada que ver con Spinoza. Y no es casual, tal y como el propio autor del artículo ha mostrado en su tesis de la mano de un interesantísimo acercamiento entre Spinoza y Michel Henry. Un desarrollo, a cargo de Sacha Carlson, sobre la crítica richiriana (desde su epojé fenomenológica hiperbólica) a una versión spinozista-henryana de la reducción fenomenológica ( $y$, por ende, a la interpretación henryana del cogito hiperbólico -o de la intepretación henryana de la filosofía de Maine de Biran-) aparecerá en breve en un próximo monográfico sobre Richir en la revista Eikasía. [NdT].

${ }^{28}$ El francés dispone, para el espacio (con la voz "ailleurs"), del equivalente semántico de lo nuestro "otrora" es para el tiempo. [NdT].

29 PIA, p. 135. 
¿Cuál es, entonces, el estatuto fenomenológico de este Genio Maligno? ya que alguna será la forma, propiamente fenomenológica, bajo la cual habrá de aparecérseme. La hipótesis seguida aquí por Richir es, desde luego, resueltamente fenomenológica, y consiste en sostener que el Genio Maligno no sería sino yo mismo o, para mayor precisión, una figura del otro (el Otro) en mí. Y en la medida en que este "Otro" se halla imaginado, será sobre el estatuto de esta imaginación sobre lo que, en primer término, tendremos que dirigir nuestra atención.

En un primer momento, podríamos pensar que esta figura del Otro en mí no soy sino yo mismo, con mi cuerpo (Leib) casi trasladado por entero al interior de la imaginación, poco más o menos como cuando imagino una escena de tristeza y que me imagino formando parte de la escena imaginada, imaginando también, por ende, mi propia tristeza ${ }^{30}$. Este "yo imaginario", que se presenta como el origen de las afecciones imaginadas, es absolutamente peculiar ya que, del todo ilocalizado e ilocalizable, adquiere todos los caracteres de una suerte de "fantasma", coextensivo con lo que, en eco a Husserl, denomina Richir Phantomleib, igualmente ilocalizado y volátil ${ }^{31}$. De este modo, cabría, pues, suponer que el Genio Maligno no fuera otra cosa que dicho Phantomleib ${ }^{32}$; cabría suponer, por lo tanto, que el Genio Maligno representa el riesgo de convertirse uno en juguete de su propia imaginación, pasando así casi enteramente del lado de la imagen. De hecho, este riesgo lo enfrentamos bajo su figura más extrema puesto que el Genio Maligno es supuestamente "omnipotente": supondríamos pues al Phantomleib capaz de autonomizarse por entero en relación

\footnotetext{
${ }^{30}$ Recojo este ejemplo de Husserl: Cfr. Hua XXIII, p. 466.

${ }^{31}$ La interpretación del Genio Maligno en términos de imaginación y de Phantomleib no se encuentra en las Méditations phénoménologiques, sino sólo en Phantasia, imagination et affectivité (2004), en que Richir llega a considerar que en toda experiencia imaginativa en tanto que distinta de la phantasia, además de las cosas figuradas en imagen, hay siempre una coloración afectiva que, sin embargo, jamás queda, a su vez, figurada, como tal, en imagen. Dichos afectos pueden tener estatutos muy diversos. Esquemáticamente, podríamos decir que o bien están efectivamente experimentados [éprouvés], o bien se hallan solamente "imaginados". En este último caso se trata, entonces, de cuasi-afectos que no están ni figurados en imagen ni experimentados, pero que "habitan" la imagen de modo esencialmente nolocalizable. Es la razón por la que no hay que confundir el Phantomleib con lo que Husserl llama también el Phantasieleib. Husserl define el Phantasieleib como el cuerpo, ciertamente indeterminado, pero implicado en toda vivencia de phantasia o de imaginación, y que se atesta por el hecho de que las cosas "phantaseadas" o imaginadas están siempre orientadas: entre los árboles que imagino, algunos están en primer plano mientras que otros ocupan el trasfondo; asimismo, este centauro que llega al galope es súbitamente atacado por un dragón que se lanza contra él según un determinado ángulo de ataque, etc. (Cfr. Hua VIII, pp. 115s). Dicho de otro modo, el Phantasieleib se halla siempre localizado, lo que precisamente no es el caso del Phantomleib, al que como fuente de los afectos imaginados, ocurre estar por doquier y al tiempo en ningún sitio definido, de suerte que los afectos imaginados parecen, ciertamente, venir como de otro lugar [d'ailleurs] (Cfr. PIA, pp. 9-46, y en especial las pp. 24ss) El Phantomleib es pues como un Otro en mí del que, por principio, no puedo tener conciencia.

32 Cfr. PIA, p. 135.
} 
al yo real y presente, con lo que cabría hablar de una verdadera escisión (Spaltung) entre ambos yoes -sabemos que, efectivamente, es lo que sucede en algunos casos de esquizofrenia aguda. ¿Se quiere decir con ello que, de la mano del Genio Maligno, transitamos de nuevo por los andurriales de la locura? No otra cosa parece sugerir Richir; de la mano de la figura del Genio Maligno nos encontramos, efectivamente, muy cerca de la locura: muy cerca sin por ello estar del todo en ella ${ }^{33}$.

Efectivamente, cumple hacer notar, en un segundo momento, que no hay verdadera escisión, en Descartes, entre el yo real y el yo imaginado del otro en mí (el Genio Maligno) en la medida en que soy siempre yo (mi yo real) quien activa y libremente se pone a imaginar al Genio Maligno. Por mucho que el yo que lleva a cabo la ficción y el yo supuestamente sorprendido en su credulidad por el Genio Maligno (al extremo de convertirse en juguete de éste) no sean idénticos, a pesar de todo queda, resiste o permanece un punto de contacto entre ambos yoes que nos impide referir, aquí, una auténtica escisión (Spaltung). De ahí que el Genio Maligno no pueda identificarse por entero con el Phantomleib, que siempre es ilocalizable y, por ende, indetectable e innombrable $^{34}$; efectivamente, no es aquí el caso: si el Genio Maligno tuviese éxito en su empresa, la de engañarme, es decir, si, en definitiva, el Genio Maligno se identificase simple y llanamente con el Phantomleib, en ese caso ni siquiera me percataría de todo ello ${ }^{35}$. De hecho, he ahí la razón por la cual el yo cartesiano no queda sumido en la locura: y es que "contrariamente a los locos, es capaz de engendrar por cuenta propia, es decir, a sabiendas, el simulacro de la locu$\mathrm{ra}^{\prime 36}$.

Conviene notar también que si, de hecho, soy capaz, en virtud del fingimiento o ficción libre y activa, de librarme de la captación por el Phantomleib y por la imaginación, es porque esta ficción no está acompañada por género alguno de reducción eidética:

Por el hecho de ser yo quien efectúa la hipérbole, y por el hecho de que ésta no concierne en absoluto a los contenidos de las "opiniones", sino a su estatuto (su pretensión a la verdad), por el hecho de que, por lo tanto, no son otros posibles contenidos

${ }^{33}$ Cfr. PIA, pp. 135s.

34 "El engaño de que el Genio Maligno sería capaz sólo sería engaño por "sorpresa" integral de mi "crédito [créance]" si el Genio Maligno operase como Phantomleib; pero entonces sería del todo indetectable, inasignable e incluso innombrable". PIA, p. 137; el subrayado es mío.

${ }^{35}$ Cfr. PIA, p. 139.

${ }^{36}$ Idem. 
de mis opiniones lo que me pongo a imaginar, no corro el riesgo de pasar a ensoñaciones en vigilia [rêveries éveillées] más o menos coherentes $y$, desde las mismas, al Phantomleib, sino que, muy al contrario, ocurre que, en cierto modo, "conjuro" este riesgo precisamente no saliendo de, o no abandonando dichos contenidos respecto de los cuales me limito, simplemente, a imaginar, por un cierto espacio de tiempo, que no son sino imaginarios. El yo que está aquí en cuestión permanece despierto y activo, y la epojé fenomenológica hiperbólica consiste, simplemente, en situar cualquier cosa que pueda pensar en régimen de "cuasi" o de "como si", es decir, en el registro "modificado" de la ficción, tratando, una vez más, de engañarme a mí mismo de forma activa dado que sé que, de hecho, no es, el que dibuja esta ficción que ahora incoo, el caso. ${ }^{37}$

Este texto es capital para comprender el sentido de la epojé fenomenológica hiperbólica, epojé cuya primera figura ${ }^{38}$ aquí examinada llamaré "reducción cartesiana"139. En primer lugar, la duda no se dirige aquí sobre los contenidos de mis "pensamientos", que hay que comprender como el conjunto de mis pensamientos, juicios, percepciones, sensaciones, imaginaciones, etc. Sin preguntarme, pues, por el contenido de mis pensamientos para cerciorarme de su legitimidad, se trata aquí de suspender, de forma mucho más global, la pretensión de mis pensamientos a la verdad: hay que ir (hiperbólicamente) hasta sospechar que pueda haber posibilidad de ilusión en el corazón mismo de mi pensar. Reparemos, pues, en la notable diferencia con Husserl, para el cual la reducción fenomenológica, en último término, precisamente ha de conducir a decantar el sentido eidético de los contenidos de mis pensamientos. De ahí que convenga hacer notar a continuación que la duda hiperbólica, por el contrario, no se ve nimbada de reducción eidética alguna: en la medida en que la duda no atañe a los contenidos de pensamiento, es imposible imaginar otros contenidos sobre la base de los contenidos que se ven sorprendidos por la duda; es, al cabo, imposible proceder a una variación eidética ${ }^{40}$. Por último, cumple subrayar que es ciertamente la imaginación el motor o la facultad de la duda cartesiana: dudar es, para Descartes, imaginar que todos mis pensamientos son... iimaginarios! La modalidad de la imaginación puesta aquí en juego es, efectivamente, del todo peculiar puesto que, por un lado, no se refiere ésta a los contenidos de

\footnotetext{
37 PIA, p. 136.

38 Dejamos el examen de otras figuras para trabajos ulteriores.

39 Se notará que, en un primer término, Richir parece identificar la duda hiperbólica cartesiana con su propia versión de la epojé fenomenológica hiperbólica. Veremos, con todo, como Richir terminará también por apartarse de Descartes, aunque sea en nombre del "hipercartesianismo" con el que también -y acaso tanto más- se apartará, como veremos enseguida, de Heidegger.

${ }^{40}$ Lo cual no está sino implícitamente sugerido en el texto que comento aquí. Richir lo escribe más explícitamente más adelante: "la epojé hiperbólica cartesiana no supone que su propia ejecución vaya, por así decirlo, de la mano o a modo de complemento de una reducción eidética". PIA, pp. 135s.
} 
pensamiento a partir de los cuales podría yo, fácilmente, montarme un mundo imaginario coherente hasta pasar por completo del lado de la imaginación (caso del Phantomleib), volviéndome, por ende, mero juguete de mi propia imaginación; y, por otro lado, la imaginación no se refiere aquí más que a su propio poder: imaginar un Genio Maligno equivale, en un contexto fenomenológico, a imaginar la captura ilusionante del yo por la imaginación, equivale, en resumidas cuentas, y como hemos sugerido, a imaginar que estoy loco. En la medida en que la imaginación es aquella facultad que puede tanto poner en imagen como generar ilusiones ${ }^{41}$, será la propia imaginación y ella sola la que pueda, "viéndoselas" consigo misma, desbaratar las trampas de la engañifa y de la ilusión.

Tras el episodio del Genio Maligno, Descartes encadena su argumento, en su segunda Meditación, para hallar en el cogito el añorado "punto arquimédi$\mathrm{Co}^{\prime \prime 2}$. Me limitaré a recordar el pasaje en el que todo parece jugarse, y que ha sido objeto de comentario, poco menos que al infinito:

\begin{abstract}
¿Acaso no soy también yo quien trataba de convencerse de no ser? Mas, sin duda, era mientras me convencía o siquiera ya por el hecho de pensar algo. Pero he que hay no sé qué engañador muy poderoso y astuto que emplea toda su industria en engañarme de continuo. No cabe duda de que soy si me engaña: podrá engañarme tanto como quiera, que jamás podrá arreglárselas para que no sea nada en tanto piense yo ser algo. De suerte que, tras haber meditado y considerado minuciosamente todas estas cuestiones, se ha de concluir y tener por permanente la siguiente proposición: que yo soy, yo existo, es necesariamente verdadera todas y cada una de las veces en que la pronuncio $o$ en que la concibo en mi mente. ${ }^{43}$
\end{abstract}

¿Qué es, pues, lo que de la duda se desprende? Simplemente la certeza de que yo soy, de que yo existo: ego sum, ego existo.

En el marco de la lectura fenomenológica de Richir, que distingue entre dos yoes, el yo que lleva a cabo la ficción, y el yo imaginario, supuestamente embaucado, la primera cuestión que se plantea es, claro está, la de saber de qué yo, de qué ego se trata aquí. Se trata, en efecto, del yo concreto, del yo del filósofo que, meditando, lleva a cabo la ficción ${ }^{44}$. Es él lo único que se desprende de la duda hiperbólica, revelándose, en virtud de mi astucia, irreductible a toda astucia del Genio Maligno, del gran engañador. Lo que se desprende de la

\footnotetext{
${ }^{41}$ Cfr. PIA, p. 138.

42 Cfr. MM, p. 19.

${ }^{43}$ Idem.

${ }^{44}$ Cfr. PIA, p. 140.
} 
hipérbole es, finalmente, mi existencia en la desnudez de su enigma; o también -diré anticipando- el Dasein, e incluso el Dasein en su facticidad ${ }^{45}$.

Otra forma de considerar el cogito estribaría en ver en ello una suerte de apercepción transcendental inmediata. Richir se explica al respecto en el siguiente texto:

Mientras finjo (imagino) activamente, mientras ejecuto la epojé hiperbólica [...] estoy despierto, pero esta mi vigilia puede tornarse, de modo instantáneo, en sueño, y mi ensoñación [rêverie] en una suerte de equivalente de un "estado hipnótico" si quedo preso, capturado, en la ilusión que la ficción vehicula consigo [...], y según la cual mi pensar, todo mi pensar, sería, en última instancia, el pensar del Otro, del propio Genio Maligno. ${ }^{46}$

La apercepción transcendental ${ }^{47}$ es ese estado -estado de lo más común pero quizá por ello extremadamente difícil de describir- en el que "sé" que yo soy yo y no otro, que estoy despierto (o incluso que duermo) sin que me haga falta pensar en ello (o "pellizcarme") para saberlo ${ }^{48}$. Este estado suele acompañar mis representaciones sin que sea explícitamente tematizado u objeto de reflexión. Sin embargo, siempre es posible ejecutar la reflexión que permite, de modo exclusivo, atestar de modo relativamente aislado o exento, la apercepción; reflexión a raíz de la cual comprendemos que la apercepción es, efectivamente, primigenia, y que comporta en sí misma la posibilidad de su propia reflexión. Se advertirá que, efectivamente, es eso lo que ocurre con el cogito cartesiano: el "yo soy, yo existo" atesta, mediante la operación reflexiva ${ }^{49}$ de la ficción, la apercepción transcendental, es decir, el hecho de que yo soy yo y de que estoy despierto. No obstante, Richir precisa de inmediato que es siempre posible perder la supuesta continuidad de la vigilia (apercepción transcendental): y ése es precisamente el caso siempre que me dejo capturar por mi ima-

\footnotetext{
${ }^{45}$ Y por lo demás, es aquí también donde se juega la institución del sujeto de la modernidad, tal y como Richir lo explica pormenorizadamente en su artículo: "Doute hyperbolique et machiavélisme. Institution du sujet moderne chez Descartes".pdf.

${ }^{46}$ PIA, p. 140.

${ }^{47}$... Y que corresponde a lo que Richir llama a veces la apercepción transcendental "en su sentido corriente" (por ejemplo, en su obra L'institution de l'idéalité. Des schématismes phénoménologiques, Beauvois, Mémoires des Annales de Phénoménologie, 2002) citado "IID", p. 34), para distinguirla de un modo más arcaico de la apercepción que trataremos con más pormenor en algunos trabajos afines a éste. Sobre este punto ver también el imponente artículo: "L'aperception transcendantale immédiate et sa décomposition en phénoménologie". pdf, Revista de Filosofía de la Universidad Complutense de Madrid (2001).

${ }^{48}$ Cfr., por ejemplo, IID, pp. 48ss.

${ }^{49}$ Por retomar los términos de los citados artículos de Pablo Posada Varela (Cfr. nota 2, in supra), operación "hiperactiva" por la que se desencadena la hiperpasividad de la hipérbole. Se trata de los dos términos de lo que Pablo Posada Varela llama "cinestesia fenomenologízante", cfr. sobre todo: "Concrétudes en concrescences. Éléments pour une approche méréologique de la réduction phénoménologique et de l'époché hyperbolique", Annales de Phénoménologie 10 (2011).
} 
ginación, siempre que ingreso en la hipnosis del Phantomleib, experiencia que presenta, en la esquizofrenia, un caso extremo.

Cabría entonces pensar que el cogito tan sólo desemboca en la existencia desnuda y desencarnada. Efectivamente, conviene insistir en ello: si bien el cogito me asegura de que pienso y de que soy, no me enseña aún ni qué pienso ni quién soy. Ya lo hemos referido: la duda cartesiana no tiene alcance sobre el contenido de mis pensamientos sino sólo sobre su valor de verdad. De hecho, es precisamente éste el lugar del desenlace de la trama cartesiana, y que Richir sigue explicitando como sigue:

"Lo que resulta de la hipérbole no son los contenidos de mis pensamientos, siempre susceptibles de engaño y de ilusión en la medida en que son imaginables (figurables en imágenes), sino el yo como lugar de la actividad misma del pensar, y del que jamás cabrá imagen. De ese modo, si en la punta extrema del cogito no sé aún quién soy, al menos me sé ya no ser "nada de lo que pudiera fingir e imaginar". ${ }^{50}$

De ahí que "lo que surge en el cogito escape, al cabo, a la ficción o a la imaginación por resultar intuitivamente infigurable en y por imaginación" ${ }^{\prime 51}$.

Pero entonces, ¿qué soy y a qué se refiere exactamente la certeza cartesiana? Precisamente a lo que soy: "una cosa que piensa (res cogitans)"52, responde Descartes, y bien sabemos cuán a menudo se le habrá reprochado dicha expresión. Richir considera, empero, que el cogito no desemboca en absoluto, contra lo que porfía la cansina cantinela de Heidegger en este punto, en un yo cosificado, exangüe o desencarnado. El ego que se descubre en el cogito resultante de la duda hiperbólica no es la substancia o el ente disponible (vorhanden $)^{53}$ sobre los que tanto insiste la interpretación heideggeriana, interpretación por desgracia de antemano autorizada a pesar de ser algo fácil o incluso zafia (cuando no, simplemente, errada). En aras a comprender la intrincada sutileza de este cogito, es preciso leer a Descartes de cerca: "¿Qué es una cosa que piensa? Es una cosa que duda, que concibe, que afirma, que niega, que quiere, que no quiere, que también imagina y que siente ${ }^{\prime \prime 54}$. En la medida en

50 MM, p. 21.

${ }^{51}$ PIA, p. 142.

52 MM, p. 22.

${ }^{53} \mathrm{Cfr}$. "Doute hyperbolique et machiavélisme. Institution du sujet moderne chez Descartes".pdf, $\mathrm{p}$. 115.

${ }^{54}$ MM, p. 22, subrayado por Richir (in PIA, p. 142). Sobre este pasaje de las Meditaciones ya había llamado la atención el propio Husserl: "Sabemos que Descartes lo entendía [scil. le cogito] en un sentido 
que en el cogito el pensar se nota [s'aperçoit] en todo su carácter incoativo, incluso más acá de la distinción entre sentir y conocer, cabe asegurar que la res cogitans es considerada, por Descartes, en la entera contingencia de su concreción, que es también la de su carne [chair]:

a fuer de haber glosado en exceso respecto del dualismo cartesiano, acaso no se haya subrayado lo suficiente que el cogito es, en Descartes, el impensable momento de la encarnación, y con cuya facticidad topamos de nuevo bajo la forma de la incomprensible contingencia de la "unión substancial" del alma y del cuerpo, momento cuya reconstrucción metafísica en términos de "glándula pineal" habrá de resultar forzosamente inadecuada. ${ }^{55}$

De todo ello tenemos que quedarnos, en esencia, con que el cogito sí se presenta, en Descartes, como auténtico momento fenomenológico, del mismo modo que la duda hiperbólica se torna, al menos "implícitamente" ${ }^{56}$, como epojé fenomenológica. Por esa vía cabe, efectivamente, una aproximación entre Descartes y Husserl; mas contrariamente a lo que creía este último, la duda cartesiana se aventura a bastante mayor profundidad, en las honduras del humano vivir ${ }^{57}$, de la que jamás osó sondar el padre de la fenomenología. Richir describe la epojé o la reducción cartesiana como sigue:

Epojé en la que, sencillamente, los "objetos" (intencionales) respectivos quedan puestos fuera de curso (pueden, efectivamente, ser figurados por la imaginación) mediante lo que les corresponde en la cogitatio (en términos husserlianos: las vivencias; no pueden ser percibidas), y que es, por lo que a ello hace, intuitivamente infigurable. [...] Comprendemos todo lo que esta vivencia, por así decirlo en su cara subjetiva, hilética y noética, conlleva de inasible (de puro infigurable), y ello siempre que dicha vivencia, en su cara subjetiva, no se halle ya de antemano apresada y estructurada como polo de la correlación noético-noemática en su sentido husserliano. Dicho de otro modo, siempre que la intencionalidad haya sido hiperbólicamente puesta fuera de curso, lo cual, a nuestro parecer, se ha de hacer si es que se quiere acceder al registro más arcaico de la fenomenología y, precisamente, es ello lo que nos lleva a reivindicar esa parte, a nuestro parecer la más importante, de la herencia cartesiana. ${ }^{58}$

Conviene recalcar que lo que, en este nivel, separa a Husserl de Descartes, reside en que, contrariamente al primero, Descartes, al no implicar los contenidos de los pensamientos en su "reducción", consigue aproximarse a las viven-

tan amplio que en él incluía todo aquello a que se refieren las fórmulas: 'percibo, me acuerdo, imagino, juzgo, siento, deseo, quiero' así como todas las demás vivencias subjetivas semejantes, con sus innombrables y fluyentes ramificaciones" (Ideen I, § 34, pp. 61s).

${ }^{55} \mathrm{MP}, \mathrm{pp} .80 \mathrm{~s}$.

${ }^{56}$ Cfr. PIA, pp. 132 y 143.

57 O "Galerías del alma" que diría Antonio Machado, del que Richir es un incansable y acérrimo lector, amén de profundo admirador. [NdT]

58 PIA, p. 143. 
cias con anterioridad a sus estructuraciones objetivas intencionales. Dicho de otro modo, Descartes es, en este punto, bastante más radical que Husserl en la medida en que pone fuera de juego la propia intencionalidad, es decir, también la eidética, a pesar de que esto último no se halle sino implícito en el texto citado. De ese modo, las vivencias se manifiestan en su naturaleza aún incoativa, infinita y salvaje, que es precisamente lo que Richir caracteriza como el registro fenomenológico "más arcaico".

Al reivindicar esta parte de la herencia cartesiana, Richir es, claro está, consciente de que retoma, solamente, un único momento. Sabemos, por caso, que la continuación del texto de las Meditaciones metafísicas, buscando asegurar el conocimiento objetivo, se dedicará a recubrir metódicamente esta caja de Pandora abierta por la hipérbole. Richir ha comentado también la continuación del texto de las Meditaciones. Sin embargo, en la medida en que, en esa parte del comentario richiriano, la cuestión de la epojé no es ya directriz, me limitaré a dos observaciones al respecto:

En primer lugar, es evidente que la segunda parte de la segunda Meditación, con el célebre análisis del trozo de cera, se encuentra en "radical ruptura" $^{\prime 59}$ con lo que precede, pues se trata, en definitiva, de hallar, sobre el fondo de la certeza del Yo soy, infigurable e indeterminado, la evidencia verdadera de los contenidos del pensar, de los cuales hasta entonces no se había hecho cuestión. En otras palabras, pasamos del pensamiento salvaje e incoativo, al conocimiento, que es conocimiento objetivo y mediante el cual reconozco lo que veo como siendo ${ }^{60}$. Vale decir que desde un momento fenomenológico llegamos a una construcción metafísica, siendo la razón de ello que el análisis "se ve profundamente obliterado por el uso prácticamente ingenuo de la lengua y de los conceptos filosóficos" ${ }^{\prime 1}$.

En segundo lugar, este movimiento metafísico se aderezará en la tercera Meditación, cuando, para cerciorarse no sólo de la nitidez del pensar por lo que hace a sus contenidos, sino también de su verdad, Descartes recurrirá al argumento ontológico: para estar en cierto de que de veras pienso cuando estoy pensando, acabo considerando "la idea del infinito": "la idea mediante la cual concibo un Dios soberano, eterno, infinito, inmutable, omnisciente, omnipoten-

\footnotetext{
59 "Doute hyperbolique et machiavélisme. Institution du sujet moderne chez Descartes".pdf, p. 117.

${ }^{60} \mathrm{Cfr}$. Idem.

${ }^{61}$ Idem.
} 
te, y creador universal de todas las cosas que están fuera de él: esta idea -me digo a mí mismo- ciertamente alberga en sí más realidad objetiva que todas aquellas mediante las cuales las substancias finitas me son representadas" ${ }^{\prime 62}$; y este Dios no puede ser mentiroso "ya que la luz natural nos enseña que el engaño necesariamente depende de algún tipo de defecto"63, a propósito de lo cual Richir comenta:

Dios me acoge, por lo tanto, en mi facticidad en tanto que portador, en sí mismo, en y por la estructura del argumento ontológico, del exceso mismo de la tautología simbólica entre contenido [teneur] de sentido de pensamiento y contenido de sentido de ser. Ambos contenidos de sentido se identifican simbólicamente en Dios sólo en virtud del propio exceso que torna su identidad inconcebible: he ahí, sin duda, y por siempre jamás, el enigma del argumento ontológico $y$, al tiempo, el de su naturaleza tautológica que, al ser tomada desde un punto de vista estrictamente lógico, no es sino -Kant lo ha mostrado de modo irreversible- un error o una ilusión transcendental. ${ }^{64}$

Así pues, si puedo estar seguro de que lo que pienso cuando de veras pienso verdaderamente es, ello es porque los entes (lo que es) y las Ideas (lo que es pensado) hallan su identidad y correspondencia en Dios, mientras que la propia Idea de Dios me excede y se me hace, por ende, inconcebible. De todo esto habremos de retener que el acceso a la verdad, tanto a la verdad de mi Yo -el saber verdadero de que soy- como a la del mundo, no es de naturaleza fenomenológica, sino que se sustenta en un argumento tautológico, a saber, el argumento ontológico, por el cual se "atesta" la verdad del ser y del pensar"

\section{HEIDEGGER Y LA FACTICIDAD DEL DASEIN COMO ARGUMENTO ONTOLÓGICO SUBREPTICIO}

¿Qué decir de Heidegger? Empecemos señalando que el acercamiento propuesto por Richir entre Descartes y Heidegger no descansa en absoluto en la interpretación de Descartes por parte de Heidegger. Como sabemos, al menos desde Sein und Zeit, este último reprocha al cogito sum cartesiano el haber

62 MM, p. 31.

${ }^{63} \mathrm{MM}$, p. 41.

64 MP, p. 83.

${ }^{65}$ Constataremos, asimismo, que se advierte una evolución de la lectura richiriana del recurso cartesiano a la Idea de infinito. En un primer momento, Richir parecía interpretar esta idea como la de un "infinito simbólico" ("Doute hyperbolique et machiavélisme. Institution du sujet moderne chez Descartes".pdf, p. 119), mientras que en Phantasia, imagination, affectivité, elabora un acercamiento de la experiencia cartesiana del infinito a la experiencia fenomenológica de lo sublime. Volveré más adelante sobre el particular. 
dejado "completamente indeterminado el sum, a pesar de ser éste considerado como no menos primordial que el cogito" ${ }^{66}$. Heidegger le reprocha a Descartes precisamente el haber interpretado unilateralmente el modo de ser del sum como Vorhandensein ${ }^{67}$. Sin embargo, hemos visto que para Richir esta interpretación no se sostiene. Es más bien al contrario: lo que en el cogito es entrevisto se encuentra incluso muy cerca de lo que el propio Heidegger intentó pensar como facticidad del Dasein.

¿Qué decir al respecto? Por facticidad hemos de entender, de modo general, el carácter de un hecho con su sentido, pero sin que este sentido sea reducible a una significación ${ }^{68}$. Heidegger explica que todo ente, en tanto que es, procede de un determinado estado de hechos. Por ejemplo, este vaso de vino vacío, delante de mí, procede de un determinado estado de hechos que Heidegger denomina el estar-ahí-delante-factual (tatsächlich Vorhandensein), y que es el propio de los entes disponibles (Vorhandenes). El sentido coextensivo con este hecho es entonces una significación bien calada o trabada [signification bien arrêtée]: la que en mi ejemplo designa el estado vacío (por desgracia) de esta cosa que tengo delante y que es un vaso de vino. Por el contrario, "la factualidad propia del hecho del Dasein, ese modo en el que todo Dasein es cada vez, lo denominamos su facticidad"69. Y "el concepto de facticidad comprende esto: 'el ser-en-el-mundo de un ente intramundano', pero de un ente capaz de comprender, de comprender en tanto está vinculado, en su 'destino', al ser del ente que le hace frente en el interior de su propio mundo"70. La facticidad es pues, según Heidegger, el hecho, para el Dasein, de ser en el mundo; se trata, sin embargo, de un hecho que tiene y hace sentido: no ya el sentido opaco de un hecho bruto, sino el sentido mismo del ser, irreductible a toda significación o nominación, y que habita, de ese modo, la ek-sistencia del Dasein. Por lo demás, este sentido no es sólo el sentido de aquel que está en el mundo,

\footnotetext{
${ }^{66}$ Sein und Zeit, Tübingen, Max Niemeyer Verlag, 1993 [1927], p. 46 (que citaré a partir de ahora como: SuZ).

67 Cfr. Jacques Taminiaux, op. cit., p. 233.

${ }^{68}$ El término alemán de Faktizität, y que se traduce en francés por "facticité", en castellano por "facticidad" (término que no existe ni en el francés ni en el castellano corrientes) deriva, claro está, del substantivo faktum (hecho), y no del verbo latino facere. Dicho de otro modo, la facticidad es lo característico de un hecho, y no lo que designa un objeto "hecho" en el sentido de "fabricado". La dificultad de traducción -y de comprensión- reside en que el adjetivo francés "factice" designa precisamente un objeto como "fabricado" -también en ese sentido habla a veces Descartes de idea facticia.

[NdT]: El castellano sí tiene aquí, respecto del francés, la ventaja de disponer de dos términos que precisamente marcan la distinción entre -helos aquí- "fáctico" y "facticio".

${ }^{69}$ SuZ, p. 56.

${ }^{70}$ Idem.
} 
ni tampoco el sentido del mundo, sino el sentido del ser-en-el-mundo, por lo tanto, indisociablemente, sentido del Dasein y de su mundo.

A la luz de esto último se comprenderá mejor el acercamiento obrado por Richir: el cogito cartesiano es la certeza de un hecho: el hecho del pensar como indisociable del hecho de ser. Este hecho también es coextensivo con su sentido sin que por ello sea siquiera nombrarlo, en la medida en que los contenidos de mi pensamiento se hallan invalidados por la sospecha del engaño. De ese modo, el cogito cartesiano es, efectivamente, del orden de una certeza fáctica de pensar y de existir. Haremos notar, no obstante, que el yo fáctico cartesiano es un yo encarnado, lo cual, en cambio, no parece en absoluto ser el caso en Heidegger, cuya ceguera ante el fenómeno fundamental del Leibköper fue, como sabemos, poco menos que proverbial (sin que, por lo demás, se hayan ponderado aún del todo las desastrosas repercusiones que tamaña ceguera o garrafal insensibilidad han tenido en su fenomenología). En cualquier caso, esto manifiesta también que la concepción richiriana de la facticidad es, si es que hacía falta señalarlo, rigurosamente no heideggeriana. $Y$ es que Richir busca deliberadamente un "nuevo sentido de la facticidad"71, pero que sólo poco a poco irá decantándose en estas líneas, quedando apuntado para ulteriores trabajos. Es así que lo que acabamos de explicar en punto a la facticidad partiendo de Heidegger y de Descartes no puede sino ser una definición provisional del sentido richiriano de la facticidad.

Pero aún hay más. En la medida en que el punto de partida de la Analítica del Dasein, en Sein und Zeit, es análisis del mundo circundante o ambiente (Umwelt) como mundo de las cosas y del comercio con las cosas, en la medida en que a ese mundo corresponde, en el Dasein, no tanto el sí-mismo "auténtico", cuanto un sí-mismo anonimizado por el "se [man]", cumple señalar que será sólo en la segunda sección de la obra, y al tomarse en cuenta el ser-parala-muerte, donde podamos hallar el lugar propiamente heideggeriano del cogito cartesiano $^{72}$ :

El equivalente arquitectónico del cogito cartesiano en Sein und Zeit reside, como es manifiesto, en el nuevo arranque de la analítica engendrado al tomar en cuenta el serpara-la-muerte. [...] Será la mortalidad del Dasein la que opere una suerte de epojé fenomenológica de un "estrato" mundano impropio -inauténtico- del Dasein; Dasein

71 PIA, p. 79.

72 Cfr. MP, p. 86. 
impropio, sin lugar a dudas, por rehuir el "encuentro" con la muerte en sus íntimas profundidades. Será, por así decirlo, sobre trasfondo de muerte como el Dasein se descubrirá, al tiempo como posibilidad ontológica existencial de existir en el mundo y de existir el mundo [en sentido transitivo], y como posibilidad fáctica, ya siempre cumplida, de la existencia. ${ }^{73}$

No entra en el marco de mi proyecto comentar ahora este pensamiento difícil que relata Richir. Me limitaré a hacer notar que en el contexto heideggeriano, si bien es cierto que el Dasein supuestamente se encuentra ya siempre arrojado en su facticidad, la dificultad residirá, sin embargo, en acceder a ese sentido fáctico. La situación resulta, pues, muy próxima a la de Descartes en sus Meditaciones, donde se buscaba decantar (como se decanta un vino) o despejar (como se despeja una incógnita) ese yo que ya siempre está ahí, despierto (lo que Richir nombraba la apercepción inmediata), pero que no podía ser decantado y atestado sino recurriendo a la ficción hiperbólica del Genio Maligno. Heidegger también trata de liberar el sentido fáctico de ese Dasein ya siempre arrojado y, de modo análogo, no lo logra sino recurriendo a esa suerte de "ficción" que es el ser-para-la-muerte (y es que no se trata, precisamente, de la muerte, sino de su "anticipación") a través del cual la posibilidad de mi propia imposibilidad me permite entrever mi posibilidad más propia -el símismo auténtico. En ese sentido, el ser-para-la-muerte es, efectivamente, el "Genio Maligno" heideggeriano:

El ser-para-la-muerte es pues, desde el punto de vista arquitectónico, el "Genio Maligno" heideggeriano -acaso con el mismo argumento ontológico, muy sutilmente disimulado, y que permite el paso desde la muerte como posibilidad de la pura y simple imposibilidad del Dasein a la muerte como posibilidad de la posibilidad más propia, y en ese sentido irreductiblemente mía, y donde será el sí mismo, junto a su radical enigma, lo que, en cierto modo, habrá de ser acogido por el Dasein como la facticidad de su destino, facticidad pasada a través del círculo de fuego de la resolución. ${ }^{74}$

Con el ser-para-la-muerte, Heidegger pone, pues, en suspenso las determinaciones ónticas y factuales del Dasein en aras a descubrir su sentido fáctico, ya siempre ahí, pero siempre recubierto por la impropiedad del "se (man)". La dificultad que indica Richir aquí consiste en que no está claro que esta epojé, pretendiéndose radical como se pretende, pueda liberar un Dasein auténtico y propio, es decir, perfectamente individuado en su verdad. ¿Acaso no hay, en Heidegger, un argumento ontológico disimulado por cuanto el Dasein, que su-

\footnotetext{
${ }^{73}$ Idem.

${ }^{74}$ MP, p. 87.
} 
puestamente ha de resultar del estar resuelto ante el ser-para-la-muerte, se supone, de antemano, "verdadero"? Ahora bien, como se echó de ver con ocasión de Descartes, la verdad del ser y del pensar no se sustenta sino en un argumento ontológico ${ }^{75}$. Es esto lo que me lleva a considerar la crítica que Richir formula a propósito de la que he denominado "reducción cartesiana" como incluyendo también a Heidegger. Este diagnóstico quedará más claro en las líneas siguientes, en que se avanzará, sin desarrollarla del todo, la idea richiriana de la epojé fenomenológica hiperbólica.

\title{
3. HACIA LA EPOJÉ FENOMENOLÓGICA HIPERBÓLICA COMO REELABORACIÓN (NO HEIDEGGERIANA) DEL SENTIDO DE LA FACTICIDAD
}

Ni Heidegger ni Descartes han llevado la hipérbole lo suficientemente lejos: he ahí, finalmente, la posición de Richir por lo que hace a la que he llamado "reducción cartesiana". Y es que tanto el uno como el otro permanecen presos de una circularidad que Richir denomina "tautología simbólica", y con arreglo a la cual ya siempre hay pre-determinación del ser y del pensar. Ya no se trata aquí, claro está, de una pre-determinación intencional y eidética de los contenidos de pensamiento, tal y como ocurría con Husserl; se trata, antes bien, de que la pre-determinación se refiere al ser y al pensar como tales y en sí mis$\operatorname{mos}^{76}$. Lo más sencillo, por ahora, será tratar de explicitar el meollo de esta tesitura a partir de Descartes:

\begin{abstract}
Para saber que pienso, hace falta que sepa de antemano qué es pensar, o hace falta, al menos, tal y como Descartes lo sugiere, que deje de pensar para reconocerme como integrando, "cada vez", el pensar. Del mismo modo, para saber que existo, he de saber de antemano qué es existir, o al menos hace falta que cese, por un instante, 0 acaso por periodos (por ejemplo en el sueño) de existir para así poder reconocerme como existiendo. Finalmente, para saber que, pensando, existo, hace falta -lo que ofrece una apariencia de silogismo que el propio Descartes denunció como interpretación errónea- que ya sepa que no puedo pensar sin, de un modo u otro, existir; hace falta, por decirlo de otra forma, que la facticidad del "yo soy, yo existo" se haya abandonado a sí misma para reencontrarse de nuevas. ${ }^{77}$
\end{abstract}

\footnotetext{
${ }^{75}$ Vuelvo con más pormenor sobre la crítica richiriana al pensamiento heideggeriano del ser-para-lamuerte en el último capítulo de mi tesis doctoral, dedicada a la fenomenología de Marc Richir, y de inminente defensa.

${ }^{76}$ Richir se refiere también a una "tautología simbólica genialmente desplazada". MP, p. 93.

77 MP, p. 90.
} 
La circularidad reside, por lo tanto, en que para poder decir: "yo pienso, yo existo" es preciso saber ya (incluso en la extrema punta de la hipérbole) lo que, en cierto modo, son de veras "ser" y "pensar":

Incluso tomado en su carácter incoativo y en su indistinción, el "yo pienso" nunca está asegurado puesto que siempre hay una parte ciega del pensar que se hace difícil de atribuir simple y llanamente al pensar sin que, en el trance, se ilusione uno en punto a la "naturaleza" misma del pensar. ${ }^{78}$

Así, Descartes capta ciertamente el pensar en ese estado aún salvaje en el que éste se confunde con el puro sentir o con el soñar. Sin embargo, aquello que, no obstante, está, pese a todo, predeterminado, es el hecho de que el pensar siempre es considerado como mi pensar: "es evidente que soy yo quien duda, comprende, desea $[\ldots]^{\prime \prime 79}$. La hipérbole cartesiana es ciertamente profundísima; y Descartes llega incluso a considerar la ilusión del pensar: "Mas se me dirá que estas apariencias son falsas y que estoy durmiendo". Quizá, efectivamente, sucede que creo estar sintiendo todas estas cosas -en definitiva, que sencillamente creo estar pensando.

Así sea -repone Descartes-; con todo, al menos es cosa cierta que me parece (videor) que veo (videre), y que siento calor; y es eso lo que en mí, propiamente, se llama sentir, y ello, precisamente así tomado, no es otra cosa que pensar. ${ }^{80}$

El "pensar" cartesiano es pues captado en un incoatividad fenomenológica, es decir, captado como mera apariencia ${ }^{81}$. Pero la circularidad consiste en que el pensar, aún tomado como apariencia, ya siempre está pre-determinado como mío, luego remitido a un ego, aunque se trate de un ego infigurable: pensar de veras, así como ser de veras, no adviene más que cuando yo pienso y yo soy; he ahí la circularidad coextensiva con un argumento ontológico primitivo que aún encontramos en la hipérbole cartesiana, donde jamás se entrevé la posibilidad de ser engañado (de ilusionarse) radicalmente en punto al hecho mismo de pensar.

\footnotetext{
78 MP, p. 89.

79 MM, p. 22; el subrayado es mío.

${ }^{80} \mathrm{MM}$, pp. 22s.

${ }^{81}$ Cfr. PIA, p. 143.

Podríamos aquí utilizar con cierta pertinencia el término que Ricardo Sánchez Ortiz de Urbina ha puesto en uso, a saber, la antigua voz castellana de "aparencia". No hay, pues, que comprender aquí "apariencia" como la "apariencia" de una verdad o como la "apariencia de algo", sino como simple aparecer (sin por ello identificarse con el aparecer como autoaparecer, tal y como lo entiende Michel Henry). $[\mathrm{NdT}]$
} 
Lo mismo ocurre, mutatis mutandis, con Heidegger: la facticidad del Dasein, que se descubre primitivamente en la Befindlichkeit, debe atravesar el "círculo de fuego" del ser-para-la-muerte para descubrirse como sentido de ser de un Dasein auténtico cuyo destino ya siempre está sellado ${ }^{82}$. La circularidad consiste aquí en que el ser verdadero, consectario del pensar verdadero, entrevisto en la hipérbole heideggeriana, se halla ya siempre inscrito en el Dasein, entre sus posibilidades más propias. Tampoco aquí se considera de veras la posibilidad de una "ilusión ontológica" que afectase a las propias estructuras ontológico-existenciales del Dasein ${ }^{83}$.

Así, la aporía nodal de la "reducción cartesiana" estriba en que la facticidad que en ella asoma ya siempre se concibe como $\mathrm{mi}$ facticidad -como la facticidad de mi existencia (Heidegger), o de mi ser y de mi pensar (Descartes)-, y a partir de entonces será la "quintaesencia" de ese "sí-mismo", portadora de todos los sentidos verdaderos posibles, lo que cumplirá exhibir. Comprendemos, de paso, que la noción de facticidad tal y como es utilizada por Richir se antoja más compleja y ambigua de lo que en un primer momento pudiera parecer. Por un lado, cuando se reclama de Heidegger, considerando la facticidad siempre mía de un Dasein, Richir no es, ni muchísimo menos estrictamente heideggeriano ya que siempre concibe esta facticidad al modo de Descartes, con y en su carne o encarnadura. Por otro lado, en contrapunto a esta concepción cuasiheideggeriana, Richir adelanta también un nuevo sentido de la facticidad en el que el Dasein no se reconocería aún como un sí-mismo. Asimismo, conviene recordar que la definición de facticidad aquí ofrecida no es sino provisional: si la facticidad ha sido, hasta ahora, entendida como el hecho significativo o con sentido [fait sensé], pero infigurable y no reductible a significación, de la existencia encarnada del Dasein, hará falta, ahora, despejar una facticidad más arcaica, siempre, claro está, encarnada, pero en inminencia de perder su ipseidad y de no ser ya coextensiva con un cuerpo propio, para perderse así en la carne del mundo [chair du monde], de las cosas y de los otros. Sólo este senti-

\footnotetext{
82 Podríamos decir "sancionado" recuperando el uso que de la expresión hace Xavier Zubiri, precisamente al hablar de la muerte como aquel momento en que una vida quedará "sancionada" como "tal" vida, como "esa vida que fue" ( $y$ ello a pesar de no poder saberse, de modo articulado, cuál sea el "contenido" de lo que se sanciona como sido; sea como fuera -y sea cual fuere su contenido- la "sanción" ha caído tomando, el sentido de una vida, un aspecto completamente distinto, siendo enteramente distinta la expensa a la que queda, su resulta). [NdT].

${ }^{83}$ Cfr. MP, p. 93 y PIA, p. 169.
} 
do más arcaico de facticidad nos permitirá comprender la noción, capital en Richir, de interfacticidad transcendental.

Traducido del francés por Pablo Posada Varela 